Original paper

\title{
Four-dimensional carbon-ion pencil beam treatment planning comparison between robust optimization and range-adapted internal target volume for respiratory-gated liver and lung treatment
}

\author{
Takayuki Kanai ${ }^{\mathrm{a}, \mathrm{b}}$, Athena Paz ${ }^{\mathrm{a}}$, Wataru Furuichi ${ }^{\mathrm{c}}$, Ching-Sheng Liu $^{\mathrm{a}, \mathrm{d}}$, Pengbo $\mathrm{He}^{\mathrm{a}, \mathrm{e}}$, \\ Shinichiro Mori ${ }^{\text {a,"* }}$ \\ ${ }^{a}$ Research Center for Charged Particle Therapy, National Institute of Radiological Sciences, 4-9-1, Anagawa, Inage-ku, Chiba, Japan \\ ${ }^{\mathrm{b}}$ Department of Radiation Oncology, Yamagata University, Faculty of Medicine, 2-2-2, Iida-nishi, Yamagata, Japan \\ ${ }^{\mathrm{c}}$ Accelerator Engineering Corporation, 6-18-1-301, Konakadai, Inage-Ku, Chiba, Japan \\ d Department of Oncology, Taipei Veterans General Hospital, 201, Sec. 2, ShiPai Road, Taipei, Taiwan, Republic of China \\ e Institute of Modern Physics, Chinese Academy of Sciences, 509 Nanchang Road, Lanzhou, China
}

\section{A R T I C L E I N F O}

\section{Keywords:}

Carbon-ion radiotherapy

4D-robust optimization

Range-adapted internal target volume

Lung cancer

Liver cancer

\begin{abstract}
A B S T R A C T
We investigated the dose differences between robust optimization-based treatment planning (4DRO) and rangeadapted internal target volume (rITV). We used 4DCT dataset of 20 lung cancer and 20 liver cancer patients, respectively, who had been treated with respiratory-gated carbon-ion pencil beam scanning therapy. 4DRO and rITV plans were created with the same clinical target volume (CTV) and organs at risk (OAR) contours. Fourdimensional dose distribution was calculated using deformable image registration. Dose metrics (e.g. D95, V20) were analyzed. Statistical significance was assessed by the Wilcoxon signed-rank test. For the lung cases, the mean CTV-D95 value for the rITV plan (=98.5\%) was same as that for the 4DRO plan $(=98.5 \%, \mathrm{P}=0.106)$, while the mean D95 value for the CTV + setup margin contour for the rITV plan $(=98.2 \%)$ was higher than that for the 4DRO plan (95.2\%, P $<0.001)$. For the liver cases, the mean CTV-D95 value for the rITV plan $(=98.1 \%)$ was slightly lower than that for the 4DRO plan $(=98.5 \%, \mathrm{P}<0.01)$, while the mean D95 value for the CTV + setup margin contour for the rITV plan $(=98.0 \%)$ was higher than that for the 4DRO plan $(94.1 \%, \mathrm{P}<0.001)$. For the doses to the organs at risk (OARs), the ipsilateral lung-V20/liver-V20 values for the rITV plan $(=10.1 \%$ / $19.7 \%)$ was significantly higher than that for the 4 DRO plan $(=8.6 \% / 17.6, \mathrm{P}<0.001)$. Although the target coverage for 4DRO plan may be worse than that for rITV plan in the presence of the setup error, the 4DRO plan can improve OAR dose while preserving acceptable target dose coverage.
\end{abstract}

\section{Introduction}

Particle beam therapy, including proton beam and carbon-ion beam types, is becoming one of the major cancer treatment approaches $[1,2]$. Compared to proton beam therapy, carbon-ion beam therapy has a better dose conformity to the target and higher biological effectiveness [3-5]. As a result of extensive research, the newer centers now use the pencil beam scanning (PBS) technique. The pencil beam technique has been used since the 1980's for proton beam [6] and since 2009 for carbon-ion beam therapy [7]. Because a tumor is subject to respiratory motion (intrafractional motion), dose conformation within a tumor could be degraded due to intrafractional beam range variation and the interplay between the tumor motion and the scanned beam spot [8]. Although the dose degradation can be minimized by an abdominal compression technique [9], not all patients can tolerate abdominal compression due to discomfort and/or low lung function.

To prevent dose degradation due to respiratory motion, adding a margin is one of the major approaches. In case of photon beam therapy, a geometrical margin is added based on physical length to compensate the target motion to create the internal target volume (ITV) [10]. In particle beam therapy, however, intrafractional motion can cause beam range variation; therefore ITV is insufficient to compensate for range variation. To solve this problem in particle-beam therapy, rangeadapted ITV (rITV) is one of our major strategies [11].

\footnotetext{
* Corresponding author.

E-mail address: mori.shinichiro@qst.go.jp (S. Mori).
} 
We successfully initiated C-PBS therapy including the thoracoabdominal region in 2015 [12]. The rITV technique has been applied to our clinical treatment. Previous reports, however, have focused on the difference between geometrical ITV and rITV, or geometrical ITV and four-dimensional robust optimization (4DRO) [13-17]. To our knowledge, there is no study comparing the rITV plan and 4DRO plan with a large number of clinical cases, especially in carbon-ion beam therapy. It is important to compare dose distributions with these two techniques in order to make choices to optimize dose distribution.

Using extant patient imaging data, we compared four-dimensional CPBS dose distributions using a rITV with those using 4DRO for lung and liver cancer cases.

\section{Materials and methods}

\subsection{Patients and CT acquisition}

This study enrolled the data of 20 lung cancer cases and 20 liver cancer cases treated with C-PBS at our hospital. Patients were immobilized with urethane resin cushion (Moldcare ${ }^{\circledR}$, Alcare, Tokyo, Japan) and low-temperature thermoplastic shells (Shell Fitter ${ }^{\circledR}$, Kuraray Co., Ltd., Osaka, Japan). Treatments were performed with phase-correlated rescanning technique and with respiratory gating [18] by markerless tumor tracking system, which has the positional accuracy of $2.2 \mathrm{~mm}$ in Euclidean distance at 95\% confidence interval [19]. Therefore, a 2-3 $\mathrm{mm}$ setup error was added in this study including interfractional and intrafractional respiratory motion variability, patient positional uncertainty, and tracking error. Lung tumors were anatomically located in upper lung lobe for 9 patients, middle lung lobe in 1 patient, and lower lung lobe in 10 patients. Four-dimensional CT (4DCT) images were acquired with a 320 multi-detector CT (Aquilion ONE Vision, Canon Medical Systems Corp., Otawara, Japan) with a thickness of $2 \mathrm{~mm}$. CT imaging conditions were $120 \mathrm{kV}$ tube voltage, $100 \mathrm{~mA}$ tube current, 500 $\mathrm{mm} \times 500 \mathrm{~mm}$ of field of view. During 4DCT acquisition, respiration was monitored by an external surrogate system (AZ-733VI, Anzai Medical Co., Ltd., Tokyo, Japan). 4DCT images were reconstructed by re-sorting all CT data sets into ten equally spaced bins in the respiratory cycle (T00: peak inhalation, T50: around peak exhalation).

\subsection{Treatment planning}

\subsubsection{Volume of interest (VOI) definition}

All contours were drawn manually on the 4DCT at T50 by certificated radiation oncologists. For the lung cases, the CTV was generated by adding a 5-mm margin to the GTV. To assess the dose to organs at risk (OARs), ipsilateral lung, contralateral lung, spinal cord, and esophagus were delineated. For the liver cases, GTV, CTV, liver, and gastrointestinal tract near the target were delineated for dose assessment. Normal lung and normal liver contours were created by subtracting the GTV contour from the normal organ contours. CTVs for other respiratory phases except for T50 were calculated automatically by deformable image registration (DIR) integrated on two different systems: intensitybased DIR on software we designed, the 4D tool [20,21], for the rITV plan, and a hybrid intensity-based DIR [22] generated on RayStation 7 (RaySearch Laboratories AB, Stockholm, Sweden) for the 4DRO plan. A certified oncologist and medical physicist checked all VOIs for respective phases; no VOIs were modified manually. The gating window was set from mid-exhalation (T30, T40) to mid-inhalation (T60, T70). The procedures to create rITV and range-adapted PTV (rPTV) are described in Section 2.2.2. To effectively reduce the normal tissue dose around the target, we created a ring-shaped VOI between $5 \mathrm{~mm}$ and $15 \mathrm{~mm}$ away from rPTV for the rITV plan and CTV for the 4DRO plan. For the purpose of comparing the volume differences among CTV, rPTV, and geometrical PTV (gPTV), we created the gPTV by summing up the CTVs within gating window and geometrically adding $2-3 \mathrm{~mm}$ setup margin.

\subsubsection{Range adapted ITV treatment planning}

We routinely use the rITV plan with a single field uniform dose (SFUD) in our clinical treatment on XiO (Elekta AB, Stockholm, Sweden). The rITV is created for each beam angle using the 4D-tool [21]. As an example of lung treatment, the design of the rITV is illustrated in Fig. 1. CTV in the lung density region (approximately $0.3-1.0 \mathrm{~g} / \mathrm{cm}^{3}$ ) moves as a function of respiratory phase (Fig. 1a-c).

(1) The water equivalent path length (WEPL) values are calculated from the beam entrance to the proximal and distal edge of the target within the gating window. For example, on a certain ray (dotted line in Fig. 1), the minimum and maximum WEPL position are expressed as $\mathrm{P}$ and $\mathrm{Q}$ at the respective phases (Fig. 1a-c).

(2) Distal and proximal positions are selected for the maximum and minimum WEPL values as $\mathrm{Q}_{2}$ and $\mathrm{P}_{1}$, respectively, on the same ray within the gating window.

(3) Two positions are calculated as same as the maximum and minimum WEPL values on the CT image at the reference phase; Tref (Fig. 1d).

(4) This process is repeated for all rays. By doing this, the rITV is generated (blue line in Fig. 1d).

Generally, a setup margin is added to the ITV. However, this kind of margin does not consider changes in WEPL due to setup error. Therefore, in this study, rITV was expanded to create a rPTV by considering the WEPL changes. Setup error was simulated by shifting the isocenter relative to the patient's body by $2-3 \mathrm{~mm}$. For example, if the gating window is T40-T60, 12 sets of the scenarios ( $=3$ respiratory phases $\times 4$ shift directions, except the direction parallel to a beam, in which the impact of the beam shift along the beam direction can be neglected) are calculated for the rPTV generation. Maps of the maximum and minimum WEPL values in rITV in the beam's-eye view were calculated within the gating window. In the above example, 12 maximum WEPL maps and 12 minimum WEPL maps will be generated. The calculation grid size of these maps was set to the magnitude of setup error. For each pixel of maximum WEPL maps, the maximum value of each scenario was calculated, while the minimum value was calculated from the minimum WEPL maps. The volume between maximum WEPL points and minimum WEPL points was the rPTV.

After creating the rPTV, 4DCT images and VOI data were transferred as DICOM files from the $4 \mathrm{D}$ tool to the workstation for the $4 \mathrm{DRO}$ plan [23].

\subsubsection{D robust optimization ( $4 D R O)$ treatment planning}

$3 \mathrm{D}$ robust optimization (3DRO) is used to optimize dose distributions by adjusting treatment planning parameters, such as beam spot position and beam spot weight. To improve positional robustness, the 3DRO algorithm employs a minimax approach that minimizes optimization objectives in the worst-case scenario out of the various setup error scenarios [24]. Patient setup error is simulated as a three-dimensional shift of the planning CT. The basic concept of $4 \mathrm{DRO}$ is an extension of 3 DRO to the time axis using a 4DCT dataset. Internal motion scenarios can be evaluated by calculating dose distribution for each respiratory phase of 4DCT datasets. The worst-case scenario in the combination of internal motion scenarios and setup error scenarios is similarly considered by the minimax approach. Therefore, the advantage of the 4DRO over the range-adapted ITV is that the four-dimensional doses to the target and OARs can be directly considered. The CTV coverage is typically very similar to that of the range-adapted ITV. The 4DRO procedure with SFUD is explained as follows:

(1) Dose distributions within the gating window are calculated with the initial treatment parameters (Param 1$)$ and the respiratory phase with a worst-dose metric is selected (underdosage occurs in respiratory phase $\mathrm{T}_{1}$ in Fig. $2 \mathrm{a}$ ). 


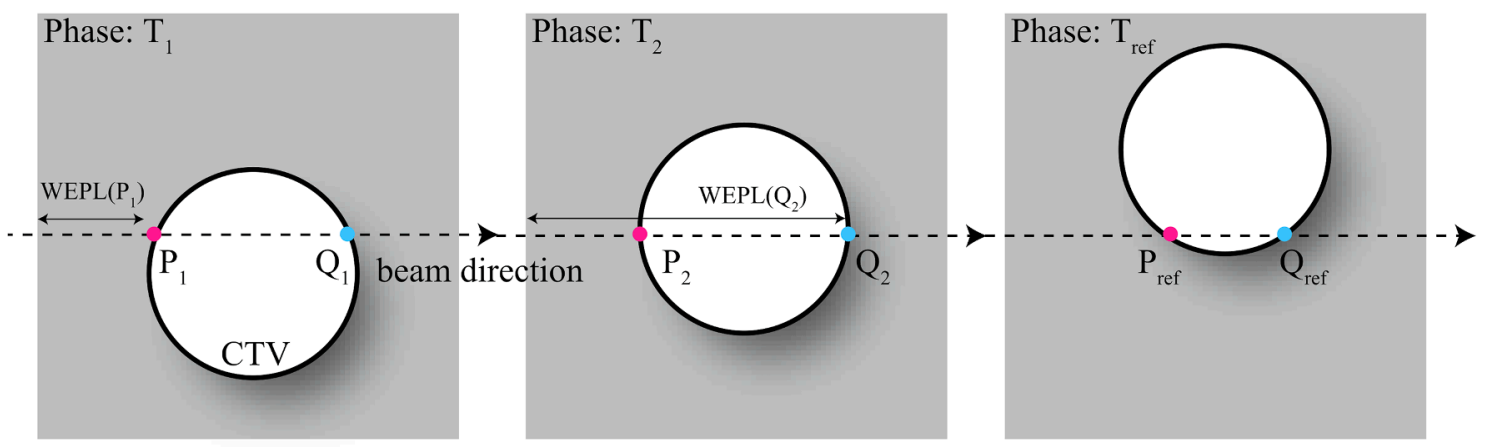

(a)

(b)

(c)

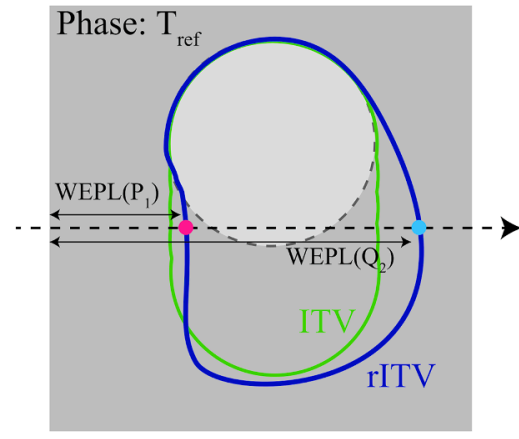

(d)

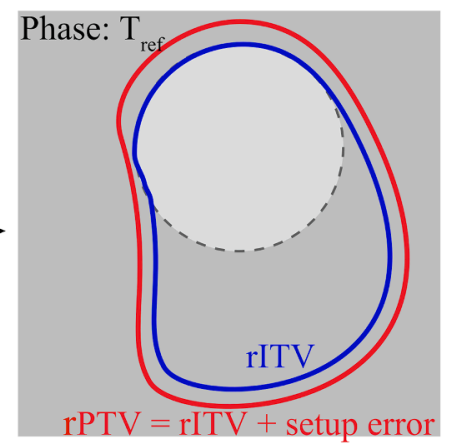

(e)

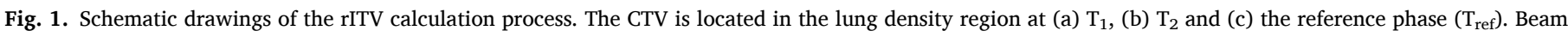

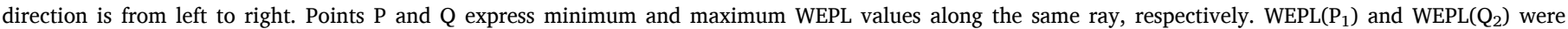

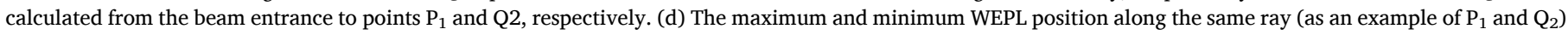

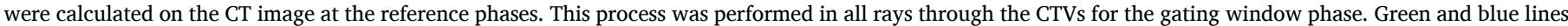

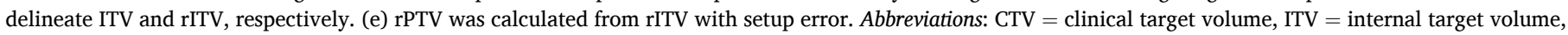

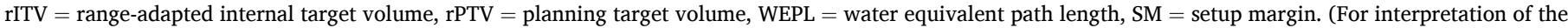
references to colour in this figure legend, the reader is referred to the web version of this article.)

(2) Treatment parameters are optimized to improve dose metrics at $\mathrm{T}_{1}\left(\right.$ Param $_{1}^{\prime}$ ) (Fig. 2b).

(3) Dose distributions within the gating window are calculated with Param $_{1}{ }^{\prime}$ and the respiratory phase with a worst-dose metric is selected (phase $\mathrm{T}_{2}$ in Fig. 2c)

(4) Treatment parameters are optimized to improve dose metrics at $\mathrm{T}_{2}\left(\operatorname{Param}_{2}\right)$ (Fig. 2d).

(5) Steps 1-4 are repeated until specified conditions are satisfied (i.e. reaching the maximum number of iterations, sufficiently low optimization objectives).

(6) As a result, high dose areas cover the CTVs in any respiratory phases. It should be noted that the setup error scenarios are ignored and only internal motion scenarios are considered for simplicity in Fig. 2.

\subsection{Dose calculation}

For the lung treatment planning, the prescribed dose of 50 Gy (RBE) in a single fraction was administered to the rPTV in ITV plans and the CTV in 4DRO plans, via four beam ports from the ipsilateral rather than the contralateral side of the tumor. For liver treatment planning, a total dose of 48 Gy (RBE) was delivered to the rPTV and the CTV in two fractions, each fraction using two orthogonal uniform fields.

For both rITV plans and 4DRO plans, dose calculations and beam spot/weight optimization with SFUD were performed on RayStation 7 (RaySearch Laboratories AB, Stockholm, Sweden). The robust optimization in RayStation employs the very similar approach to the minimax optimization, which described above. To make the optimization problem to be continuous, the maximum operator for cost functions of each scenario is replaced by the smooth power mean operator. Energy layer spacing, spot spacing, proximal target margin, distal target margin, and lateral margin were set to $2.5 \mathrm{~mm}$ (in water equivalent thickness), $2 \mathrm{~mm}$, 1 layer, 1 layer, and $0 \mathrm{~mm}$, respectively. Beam spot weights were optimized in consideration of the RBE-weighted dose distribution [25]. Optimization objectives for lung treatment planning and liver treatment planning were based on our clinical protocol and are summarized in Tables 1 and 2 .

For both treatment plans, dose distributions were calculated for the respective phases within the gating window. The four-dimensionally accumulated dose was calculated by deforming dose distribution from respective respiratory phases to the peak exhalation phase (T50) using a hybrid intensity-based DIR, as mentioned in Section 2.2.1.

\subsection{Dose assessment}

Accumulated dose distributions were evaluated using dose-volume parameters. The percent dose covering $95 \%$ of the target (D95) of the CTV was used to evaluate the target coverage. It should be noted that the dose to the CTV, instead of the rPTV, was evaluated because fourdimensionally accumulated dose distribution considers the effect from respiratory motions. However, dose evaluation for the CTV does not consider the effect by setup error. Because we have included 2-3 mm setup error in creating rITV or executing 4DRO, D95 values of the CTV + setup margin contours were additionally evaluated in order to estimate the target coverage in the presence of the setup error. The OAR volume fractions covered by 5, 10, 20, and $30 \mathrm{~Gy}$ (RBE) (V5, V10, V20, and V30, respectively) of ipsilateral lung, the maximum dose (Dmax) to the spinal cord, and the highest dose covering $2 \mathrm{cc}$ (D2cc) of the esophagus were 


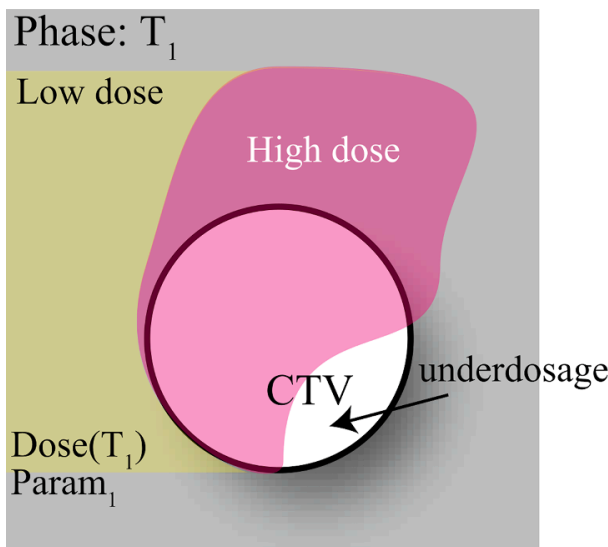

(a)

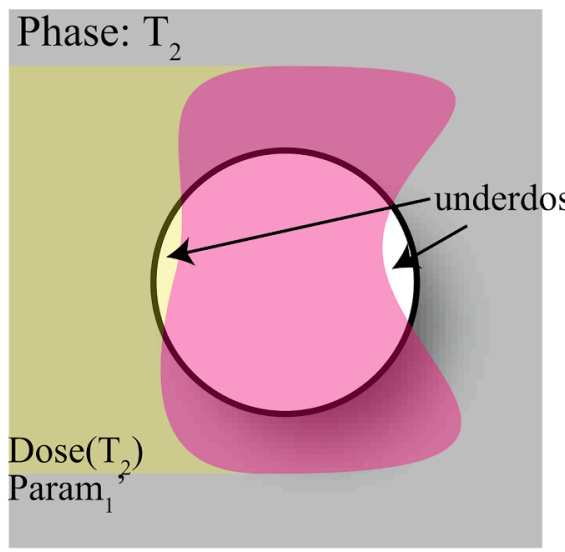

(c)

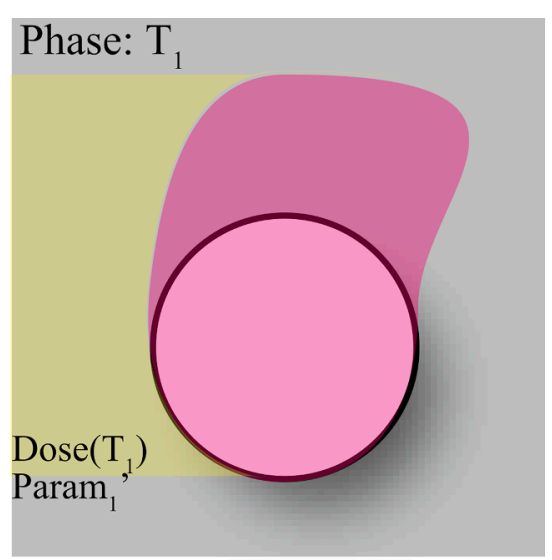

(b)

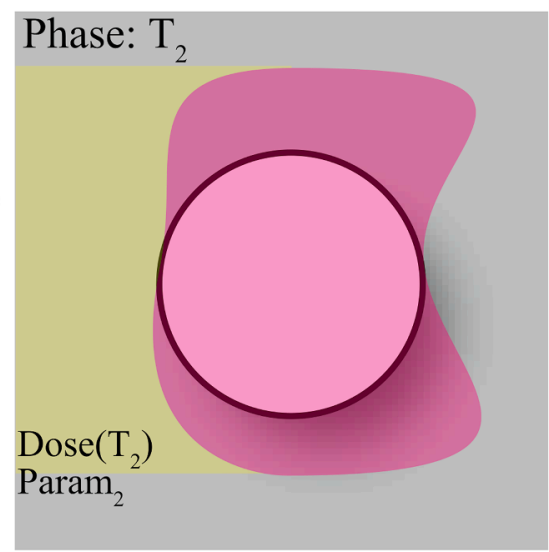

(d)
Fig. 2. Schematic drawings of the 4DRO calculation process. Beam direction is from left to right. Dose distributions (a) with the initial parameter $\left(\right.$ param $\left._{1}\right)$ at phase $\mathrm{T}_{1}$, (b) with the optimized parameter (par$\mathrm{am}_{1}{ }^{\prime}$ ) for CTV at phase $\mathrm{T}_{1}$, (c) with the param ${ }_{1}^{\prime}$ at phase $\mathrm{T}_{2}$, (d) with the optimized parameter $\left(\right.$ param $_{2}{ }^{\prime}$ ) for CTV at phase $\mathrm{T}_{2}$. CTV is within the black circle. Low and high dose regions are colored in yellow and red, respectively. Abbreviations: CTV = clinical target volume. (For interpretation of the references to colour in this figure legend, the reader is referred to the web version of this article.)
Table 1

Optimization objectives for lung treatment planning.

\begin{tabular}{|c|c|c|c|c|}
\hline $\begin{array}{l}\text { Structure (rITV plan/ } \\
\text { 4DRO plan) }\end{array}$ & Type & Dose & Weight & Robustness \\
\hline \multirow[t]{3}{*}{$\mathrm{rPTV} / \mathrm{CTV}$} & $\begin{array}{l}\text { Maximum } \\
\text { dose }\end{array}$ & $\begin{array}{l}\text { Prescribed } \\
\text { dose } \times 1.01\end{array}$ & 500 & Yes \\
\hline & $\begin{array}{l}\text { Uniform } \\
\text { dose }\end{array}$ & Prescribed dose & 100 & Yes \\
\hline & $\begin{array}{l}\text { Minimum } \\
\text { dose }\end{array}$ & $\begin{array}{l}\text { Prescribed } \\
\text { dose } \times 0.99\end{array}$ & 500 & Yes \\
\hline $\begin{array}{l}\text { rPTV-derived ring } \\
\text { ROI/CTV-derived } \\
\text { ring ROI }\end{array}$ & $\begin{array}{l}\text { Maximum } \\
\text { dose }\end{array}$ & $\begin{array}{l}\text { Prescribed } \\
\text { dose } \times 0.80\end{array}$ & 50 & Yes \\
\hline Spinal cord & $\begin{array}{l}\text { Maximum } \\
\text { dose }\end{array}$ & 10 Gy (RBE) & 100 & No \\
\hline Esophagus & $\begin{array}{l}\text { Maximum } \\
\text { dose }\end{array}$ & 10 Gy (RBE) & 100 & No \\
\hline Bronchi & $\begin{array}{l}\text { Maximum } \\
\text { dose }\end{array}$ & 20 Gy (RBE) & 100 & No \\
\hline \multirow[t]{2}{*}{ Patient body } & Dose fall-off & $\begin{array}{l}\text { High dose level: } \\
\text { Prescribed } \\
\text { dose } \times 0.95\end{array}$ & & \\
\hline & & $\begin{array}{l}\text { Low dose level: } \\
\text { Prescribed } \\
\text { dose } \times 0.50 \\
\text { Low dose } \\
\text { distance: } 1 \mathrm{~cm}\end{array}$ & 10 & No \\
\hline
\end{tabular}

Abbreviations: rITV $=$ range-adapted internal target volume, CTV $=$ clinical target volume, rPTV $=$ range-adapted planning target volume, 4 DRO $=4$ dimensional robust optimization. calculated for lung cases. V5, V10, V20, and V30 of the liver were calculated for liver cases. The dose-volume parameter difference was calculated as

Parameter difference $(\%)=\frac{X_{4 \mathrm{D}-\text { robust }}-X_{\text {rITV }}}{X_{\text {rITV }}} \times 100$,

where $\mathrm{X}_{4 \mathrm{D}-\text { robust }}$ and $\mathrm{X}_{\mathrm{rITV}}$ are the dose-volume parameters in respective plans.

Statistical significance was assessed by the Wilcoxon signed-rank test, and $P<0.05$ was considered significant.

\section{Results}

\subsection{Lung cases}

Patients' characteristics are summarized in Table 3. Tumor displacements within gating window were small (median value: $1.0 \mathrm{~mm}$ ) because about half of the tumors located the upper lobe. rPTV volumes were 2.3 times larger than CTV volumes in median value.

For the first example of a lung case (patient no. 7), tumor displacement within the gating window was $0.3 \mathrm{~mm}$ (T40-T60). Sufficient doses were received by the CTV (D95 $=98.9 \%$ for both plans), while the dose to the CTV + setup margin contour was slightly lower in 4DRO plan (D95 $=98.9 \% / 95.3 \%$ with rITV/4DRO plans). (Fig. 3a and b). Dose differences $>5 \%$ were not observed inside the CTV, while dose around the CTV was reduced with the 4DRO plan (Fig. 3c). A dose-volume histogram (DVH) showed that the 4DRO plan decreased OAR doses (ipsilateral lung V20 values (rITV/4DRO plans) $=20.5 \% / 17.5 \%$, esophagus D2cc values (rITV/4DRO plans) $=1.8 \mathrm{~Gy}(\mathrm{RBE}) / 1.4 \mathrm{~Gy}$ 
Table 2

Optimization objectives for liver treatment planning.

\begin{tabular}{|c|c|c|c|c|}
\hline $\begin{array}{l}\text { Structure (rITV plan/ } \\
\text { 4DRO plan) }\end{array}$ & Type & Dose & Weight & Robustness \\
\hline \multirow[t]{3}{*}{$\mathrm{rPTV} / \mathrm{CTV}$} & $\begin{array}{l}\text { Maximum } \\
\text { dose }\end{array}$ & $\begin{array}{l}\text { Prescribed } \\
\text { dose } \times 1.01\end{array}$ & 500 & Yes \\
\hline & $\begin{array}{l}\text { Uniform } \\
\text { dose }\end{array}$ & Prescribed dose & 100 & Yes \\
\hline & $\begin{array}{l}\text { Minimum } \\
\text { dose }\end{array}$ & $\begin{array}{l}\text { Prescribed } \\
\text { dose } \times 0.99\end{array}$ & 500 & Yes \\
\hline $\begin{array}{l}\text { rPTV-derived ring } \\
\text { ROI/CTV-derived } \\
\text { ring ROI }\end{array}$ & $\begin{array}{l}\text { Maximum } \\
\text { dose }\end{array}$ & $\begin{array}{l}\text { Prescribed } \\
\text { dose } \times 0.80\end{array}$ & 50 & Yes \\
\hline Spinal cord & $\begin{array}{l}\text { Maximum } \\
\text { dose }\end{array}$ & 10 Gy (RBE) & 100 & No \\
\hline Esophagus & $\begin{array}{l}\text { Maximum } \\
\text { dose }\end{array}$ & 10 Gy (RBE) & 100 & No \\
\hline Stomach & $\begin{array}{l}\text { Maximum } \\
\text { dose }\end{array}$ & 10 Gy (RBE) & 100 & No \\
\hline Colon & $\begin{array}{l}\text { Maximum } \\
\text { dose }\end{array}$ & 10 Gy (RBE) & 100 & No \\
\hline \multirow[t]{2}{*}{ Duodenum } & $\begin{array}{l}\text { Maximum } \\
\text { dose }\end{array}$ & 10 Gy (RBE) & 100 & No \\
\hline & & $\begin{array}{l}\text { High dose } \\
\text { level: } \\
\text { Prescribed } \\
\text { dose } \times 0.95\end{array}$ & & \\
\hline Patient body & Dose fall-off & $\begin{array}{l}\text { Low dose level: } \\
\text { Prescribed } \\
\text { dose } \times 0.50 \\
\text { Low dose } \\
\text { distance: } 1 \mathrm{~cm}\end{array}$ & 10 & No \\
\hline
\end{tabular}

Abbreviations: rITV $=$ range-adapted internal target volume, $\mathrm{CTV}=$ clinical target volume, rPTV $=$ range-adapted planning target volume, $4 \mathrm{DRO}=4$ dimensional robust optimization.

Table 3

Patient characteristics. Median values (range) are shown for age, CTV volume, rPTV volume, rPTV/CTV volume ratio, gPTV volume, respiratory cycle, duty cycle, tumor displacement. "Marginal" means tumors locating in cross-sectional part of the liver.

\begin{tabular}{lll}
\hline Characteristics & Lung case & Liver case \\
\hline Number of patients & 20 & 20 \\
Age (year) & $74(52-86)$ & $76(54-88)$ \\
CTV volume (cc) & $23.7(5.1-143.5)$ & $118.4(18.7-368.7)$ \\
rPTV volume (cc) & $55.7(14.0-325.2)$ & $184.9(33.7-552.5)$ \\
rPTV/CTV volume ratio & $2.3(1.6-3.6)$ & $1.5(1.3-2.3)$ \\
gPTV volume (cc) & $36.9(9.4-179.8)$ & $142.1(26.5-518.4)$ \\
Respiratory cycle (sec) & $3.2(2.2-5.7)$ & $3.2(2.5-4.9)$ \\
Duty cycle (\%) & $30(30-50)$ & $30(30-40)$ \\
Tumor displacement (mm) & & \\
A single respiratory cycle & $5.67(1.3-23.9)$ & $11.0(4.0-24.1)$ \\
Within gating window & $1.0(0.1-2.6)$ & $1.5(0.02-4.6)$ \\
Tumor location & & \\
& Right upper lobe: 4 & S4: 1 \\
& Right middle lobe: 1 & S7: 4 \\
& Right lower lobe: 5 & S8: 4 \\
& Left upper lobe: 5 & Marginal: 11 \\
\hline
\end{tabular}

Abbreviations: CTV = clinical target volume, $\mathrm{rPTV}=$ range-adapted planning target volume, gPTV $=$ geometrical planning target volume.

(RBE), spinal cord Dmax values (rITV/4DRO plans) =11.4 Gy (RBE)/ 9.2 Gy (RBE)) in Fig. 3d.

The DVH averaged over all lung cases were generated with mean volume values in respective dose bins (Fig. 4). Although the mean D95 value of the CTV with the rITV plan was not different from that with the 4DRO plan $(P=0.106)$, the mean D95 value of the CTV + setup margin was lower with $4 \mathrm{DRO}$ plan $(P<0.001)$. Using $4 \mathrm{DRO}$, mean values of V5, $\mathrm{V} 10, \mathrm{~V} 20, \mathrm{~V} 30$ in the ipsilateral lung, Dmax in the spinal cord, and D2cc in the esophagus were reduced by $8.7 \%, 13.0 \%, 15.3 \%, 15.6 \%, 18.2 \%$, and $23.6 \%$, respectively. The dose reduction in these OARs was statistically significant $(P<0.004)$. Dose-volume parameters for lung cancer patients are summarized in Table 4.

The dose-volume parameters for all lung cases are plotted in Fig. 5. The negative value is used to indicate a parameter value with the 4DRO plan that is lower than that with the rITV plan. Although the D95 value difference for the CTV and CTV + setup margin was small, dose volume parameters for the ipsilateral lung, spinal cord, and esophagus were significantly reduced in the most of the cases.

\subsection{Liver cases}

Tumor displacement within gating window was $1.5 \mathrm{~mm}$ in median value (Table 3). Median rPTV/CTV volume ratio was 1.5 and that was smaller than that in lung cases, because the low density in the lung tissue expanded beam ranges and resulted in large rPTV volumes.

Accumulated dose distributions (patient no.18) are shown in Fig. 6. Tumor displacement within the gating window was $0.9 \mathrm{~mm}$ (T40-T60). We found the same tendency as in the lung cases. The CTV coverage was achieved over $95 \%$ of the prescribed dose in both 4DRO and rITV plans (Fig. 6a and b), while dose coverage for CTV + setup margin was worse in 4DRO plan. The D95 values of the CTV were $98.1 \% / 98.4 \%$ with the rITV/4DRO plans and those of the CTV + setup margin contour were $98.1 \% / 94.2 \%$, respectively. The dose around the CTV was significantly reduced in the 4DRO plan, while the dose inside the CTV was almost the same (Fig. 6c). For the normal liver, V20 value with 4DRO plan was decreased from $33.0 \%$ to $29.0 \%$ (Fig. $6 \mathrm{~d}$ ).

The DVHs averaged over all liver cases are calculated in the same way as described above (Fig. 7). In comparison with the rITV plan, the dose to the CTV and CTV + setup margin was comparable and lower, respectively, with $4 \mathrm{DRO}$ plan. The liver dose was significantly reduced with the 4DRO plan. Although the mean CTV-D95 value with the 4DRO plan $(=98.5 \%)$ was slightly higher than that with the rITV plan ( $=98.1 \%, P=0.001$ ), the mean D95 value of the CTV + setup margin contour was lower with 4DRO plan $(98.0 \% / 94.1 \%$ with rITV/4DRO plan, $P<0.001$ ). Dose-volume parameters for all liver cases are summarized in Table 5.

By applying the 4DRO plan, liver-V5, -V10, -V20, and -V30 values were reduced by $8.5 \%, 9.4 \%, 10.5 \%$, and $11.2 \%$, respectively $(P<$ 0.001). Although the CTV-D95 value differences were almost zero, the dose-volume parameters (liver-V5, -V10, -V20, and -V30) were significantly reduced with the 4DRO for all patients (Fig. 8).

\subsection{Volume analysis}

To analyze how much volumes of the rPTV/gPTV increases from the CTV for each patient, we calculated volumes of the $\mathrm{rPTV} / \mathrm{gPTV}$ minus CTV. The correlations between subtracted volumes and the CTV volumes were shown in scatter plots (Fig. 9(a), (b)). It was observed that gPTV and rPTV tended to increase as the volume of CTV increased, and moderate to strong correlations were observed between them $\left(\mathrm{R}^{2}\right.$ values ranged from 0.362 to 0.903 ).

The correlations between volumes of the $\mathrm{rPTV} / \mathrm{gPTV}$ minus CTV and the residual tumor motion within gating window were also analyzed to investigate if the magnitude of tumor motion affects the volume increase of rPTV/gPTV (Fig. 9(c), (d)). As a result, the magnitude of tumor motion was poorly correlated with increased volume of rPTV and gPTV. $\left(\mathrm{R}^{2}\right.$ ranged from 0.000 to 0.141 ).

\section{Discussion}

In this study, we compared time accumulated dose distributions between the 4DRO and rITV plans. Our results showed that the dose coverage to the CTV with the 4DRO plan (mean D95 value > 98\%) was almost the same as those with the rITV plan, while dose coverage to the CTV + setup margin contour was worse in 4 DRO plan. However, 


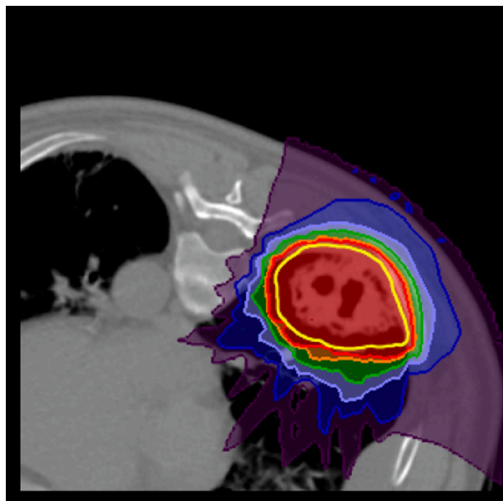

(a)
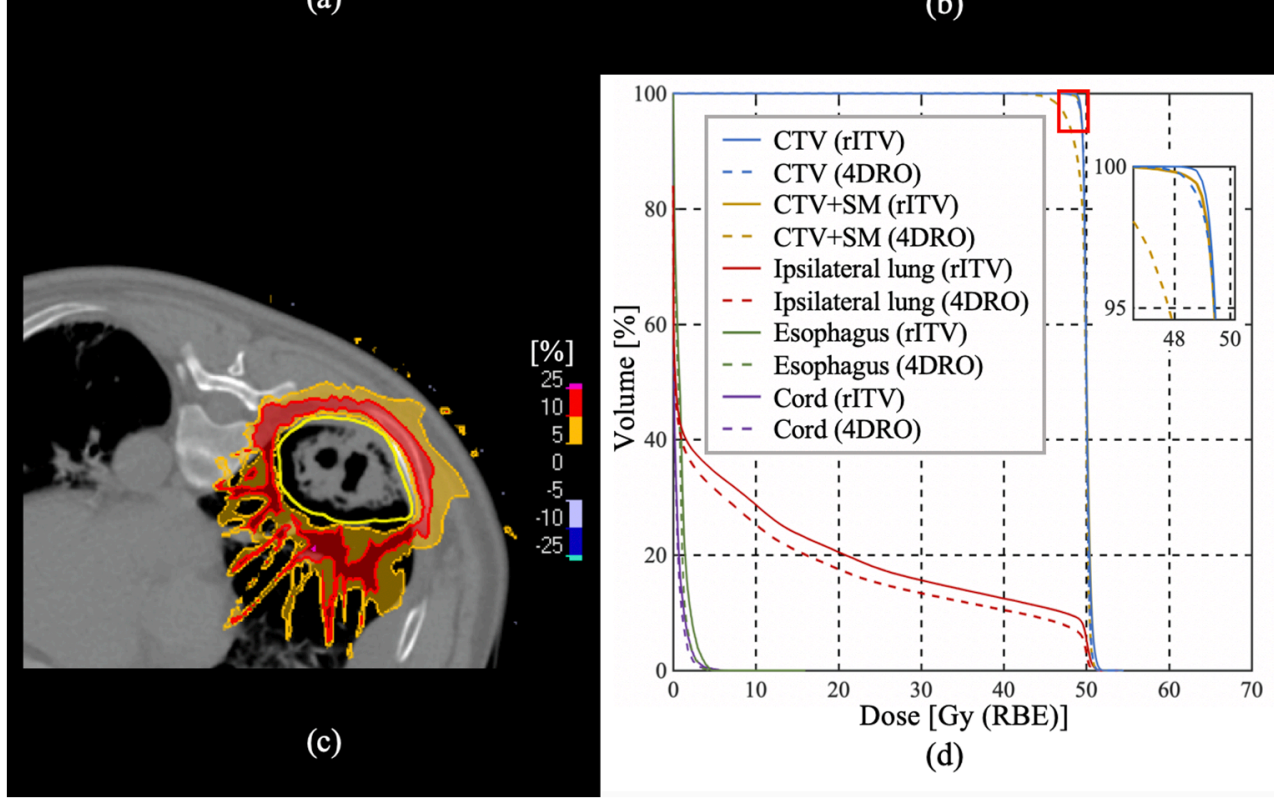

(d)
Fig. 3. Accumulated dose distributions for a lung case (patient no. 7) with (a) rITV plan, (b) 4DRO plan, and (c) dose differences (3a minus $3 b$ ). CTV is depicted as a yellow line. Red, orange, green, light blue, blue and purple lines show 95\%, 90\%, 70\%, 50\%, $30 \%$ and $10 \%$, respectively. (d) The dosevolume histograms. Because the histogram of the CTV + SM (rITV) is overlapping to other lines, the area in a red square is magnified. Abbreviations: CTV = clinical target volume, rITV $=$ range adapted internal target volume, 4DRO: 4-dimensional robust optimization. (For interpretation of the references to colour in this figure legend, the reader is referred to the web version of this article.) differences in mean D95 value were 3.1\% for lung cases and $2.4 \%$ for liver cases, respectively. It would be acceptable considering the significantly better OAR sparing was achieved by the 4DRO plan. It is remarkable that the different approaches (rITV and 4DRO) resulted in the different consequences, even if tumor displacements were small to moderate (tumor displacement ranged from 0.02 to $4.6 \mathrm{~mm}$ ).

This study mainly focused on the approaches to deal with respiratory motions. Therefore, we ignored uncertainties of stopping power calculation in order to correctly evaluate whether these approaches are effective for handling respiratory motion. However, it should be noted that this uncertainty should be considered in clinical situations.

We analyzed how much rPTV volumes and gPTV volumes are increased from the CTV, and investigated what factors cause the increase in $\mathrm{rPTV} / \mathrm{gPTV}$ volumes. Surprisingly, the magnitude of tumor motion within the gating window was not highly correlated with the increase in target volumes, although was correlated with the CTV volumes. The increase in volumes of both rPTV and gPTV depends on the shape of the tumor. Heterogeneity around the CTV will strongly affect the volume of the rPTV because the creation of the rPTV includes the calculation of WEPL. These reasons can explain the weak correlation between the magnitude of tumor motion and the volume increase in rPTV/gPTV.

Although tumor motion within the gating window was small, rPTV volumes were $1.3-3.6$ times larger than CTV volumes, this is because of the setup error and the respiratory motion (Table 3). Moreover, rPTV was still larger than gPTV due to the respiratory induced range variation. Even though the respiratory motion was small, it is important to evaluate range variation, and, if necessary, it should be considered into the treatment planning.

One of the advantages in this study is to have simulated the realistic clinical situation. Actually, the rITV approach with $2-3 \mathrm{~mm}$ setup error have been already used in our hospital. Moreover, fluoroscopic based markerless gating strategy was also implemented, therefore, intrafractional and interfractional tumor positional uncertainties including baseline shift could be minimized. By applying both strategies, we have already integrated rITV approach into the clinic [19].

\subsection{Difference between rITV plan and $4 D R O$ plan}

The 4DRO plan decreased doses around the tumor more than the rITV plan. This is explained by the following: the 4DRO procedure optimized beam spots and calculated the dose distributions in each respiratory phase. In addition, beam spots at a certain respiratory phase in a worst-case scenario were re-optimized to improve dose distribution. This process was repeated until dose distributions within the gating window met the stopping criteria. On the other hand, rITV is created by taking maximum and minimum range position to calculate where to deposit high dose, in order to compensate for range fluctuations due to respiratory motion. Therefore, rITV could overestimate the extent where a high dose should be deposited to obtain enough target coverage. Additionally, since dose optimization in an rITV plan only considers the dose distribution in a reference respiratory phase, dose perturbation in the other respiratory phases is not accurately estimated.

As favorable dose reduction to normal tissue adjacent to the target was found, reduction of radiation toxicity is expected using 4DRO. 


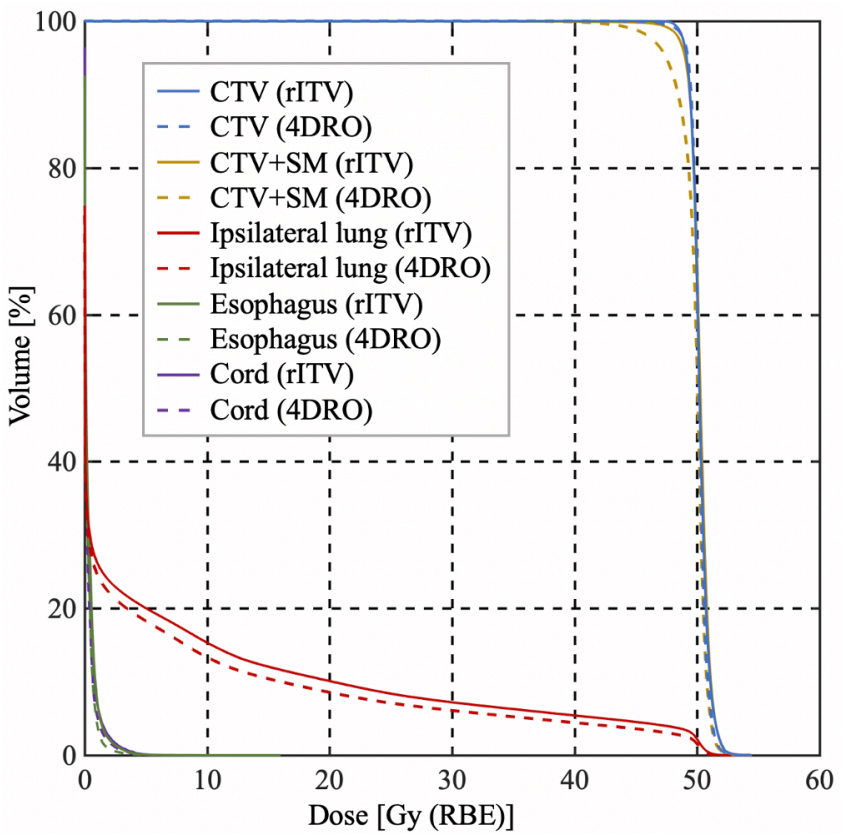

Fig. 4. Dose-volume histogram averaged over all lung cases for rITV (solid line) and 4DRO (dotted line) plans. Abbreviations: CTV = clinical target volume, rITV $=$ range adapted internal target volume, 4DRO: 4-dimensional robust optimization.

Table 4

Summary of the dose-volume parameters (Mean \pm SD) for lung cases.

\begin{tabular}{llllr}
\hline Structure & Metric & rITV plan (\%) & 4DRO plan (\%) & $P$-value* \\
\hline CTV & D95 & $98.5 \pm 0.8$ & $98.5 \pm 1.3$ & 0.106 \\
CTV + setup margin & D95 & $98.2 \pm 1.1$ & $95.2 \pm 1.8$ & $<0.001$ \\
Ipsilateral lung & V5 & $20.0 \pm 6.5$ & $18.3 \pm 6.5$ & $<0.001$ \\
& V10 & $15.3 \pm 6.1$ & $13.3 \pm 5.7$ & $<0.001$ \\
& V20 & $10.1 \pm 5.0$ & $8.6 \pm 4.6$ & $<0.001$ \\
Spinal cord & V30 & $7.5 \pm 4.2$ & $6.3 \pm 3.7$ & $<0.001$ \\
Esophagus & Dmax & $7.4 \pm 9.3$ & $6.1 \pm 8.0$ & $<0.001$ \\
\hline
\end{tabular}

*Wilcoxon signed rank test.

Abbreviations: CTV = clinical target volume, rITV $=$ range-adapted internal target volume, $4 \mathrm{DRO}=4$-dimensional robust optimization, $\mathrm{SD}=$ standard deviation.

Several researchers have reported on the significant correlation between radiation pneumonitis and dose-volume parameters, such as V20 [26-28]. It also known that there is significant correlation between liver toxicity (radiation-induced liver disease and decline of liver function) and dose-volume parameters $[29,30]$. Although radiation pneumonitis and liver toxicity are relatively mild in carbon-ion beam therapy $[31,32]$, it is reported that the risk of radiation pneumonitis increases in patients with low lung function [26]. Excessive dose reduction to the normal tissue is therefore welcome, especially in dose escalation and hypo-fractionation treatments.

On the other hand, the dose reduction around the target by 4DRO might create a risk of the under-dosage to the CTV in the presence of the setup error. Underestimation of the setup error and/or internal error will also be a risk of the unexpected under-dosage to the CTV, especially with 4DRO. To minimize these problems, sufficient setup error and internal error should be considered to allow for robust optimization.

\subsection{Effect of heterogeneity on the results}

We enrolled lung and liver cancer patient data in this study because they have different characteristics in terms of tissue heterogeneity. Lung

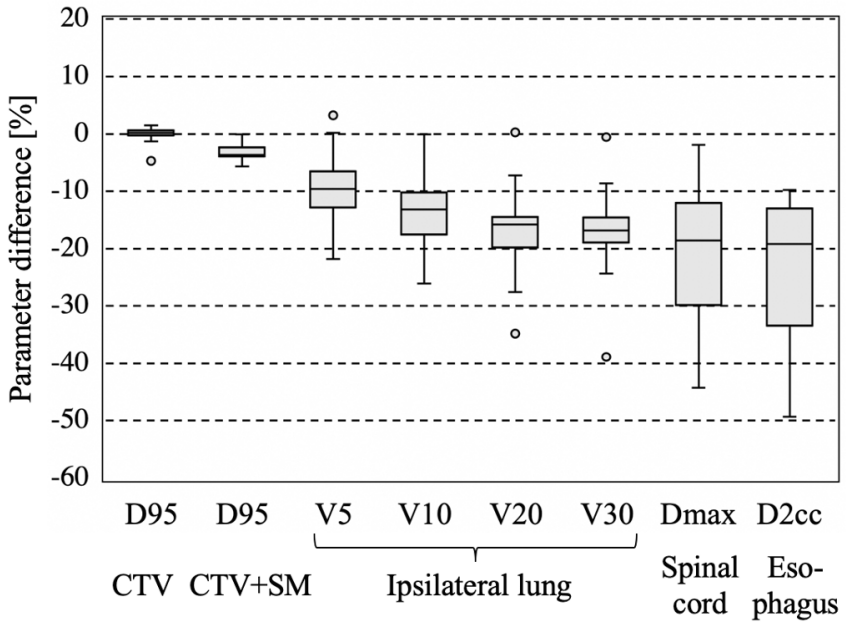

Fig. 5. A boxplot of the dose-volume parameter differences between 4DRO plans and rITV plans in respective VOIs for lung cases. Parameter differences were calculated according to the Eq. (1). The central horizontal line in the box indicates the median, and the bottom and top edges of the box indicate the 25th percentiles ( $q 1$ ) and 75th percentiles ( $q 3$ ), respectively. The whiskers extend to the most extreme data points not considered outliers. Outlier (gray circle) is identified if it is $>q 3+(q 3-q 1) \times 1.5$ or $<q 1-(q 3-q 1) \times 1.5$. Abbreviations: $\mathrm{CTV}=$ clinical target volume, $\mathrm{VOI}=$ volume of interest, $\mathrm{SM}=$ setup margin.

cancer has rather heterogeneous tissue distribution, while liver cancer has more homogeneous tissue distribution around the tumor. However, we found that 4DRO could effectively spare the normal tissue around lung and liver tumors regardless of tissue heterogeneity along the beam path. The application of 4DRO to other tumors subject to motion, such as pancreatic cancer, may similarly result in good OAR sparing and improved target coverage. This is partially supported by a previous study reporting the effectiveness of 3DRO in treating pancreatic cancer [33].

\subsection{Relevant studies}

Knopf et al. [11] reported that the use of a geometrical ITV deteriorated the target coverage in comparison with rITV. They applied fourdimensional dose calculations to both ITV-based planning and rITVbased planning and have shown the effectiveness of rITV. The effectiveness of robust optimization was also reported by Steitz et al. [33]. They compared dose distributions with four planning techniques; 3DRO, conventional intensity-modulated carbon ion therapy, SFUD optimization, and daily re-planning. In comparison with the SFUD optimization, robust optimization resulted in a lower mean dose to the kidneys of approximately $2 \mathrm{~Gy}$ (RBE), and lower near-maximum dose to the spinal cord of approximately $4 \mathrm{~Gy}$ (RBE). We found that the 4DRO-based planning resulted in a lower maximum dose to the spinal cord by 1.3 Gy and lower V20 to the lung/liver by $2.0 \% / 2.1 \%$, compared to rITV planning. Therefore, 4DRO has consistently resulted in better normal tissue sparing than the other techniques.

Lin et al. [34,35] reported the beam specific PTV with flexible smearing improved organ sparing and target coverage, in comparison with fixed smearing approach. The flexible smearing technique uses maximum and minimum intensity projection (MIP and MinIP) of 4DCT and average CT images. This technique is similar to rTV technique in terms of using 4DCT and taking maximum and minimum WEPL. However, MIP/MinIP-based calculation of maximum/minimum WEPL can overestimate the beam-specific PTV, because the MIP/MinIP-based WEPL is always larger/smaller than that in any respiratory phase of 4DCT images. Therefore, flexible smearing approach is more conservative in keeping target coverage than rITV approach.

Although we compared two treatment planning techniques for 
(a)

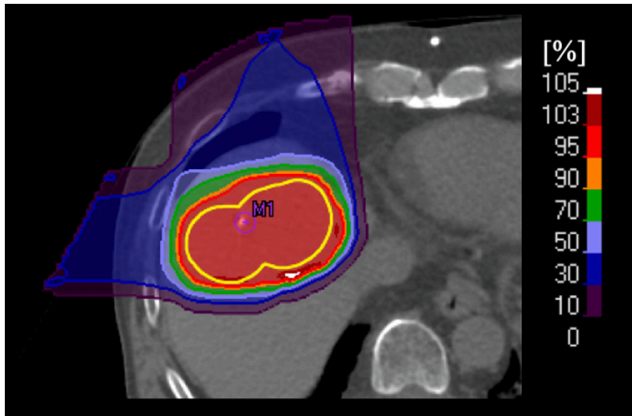

,
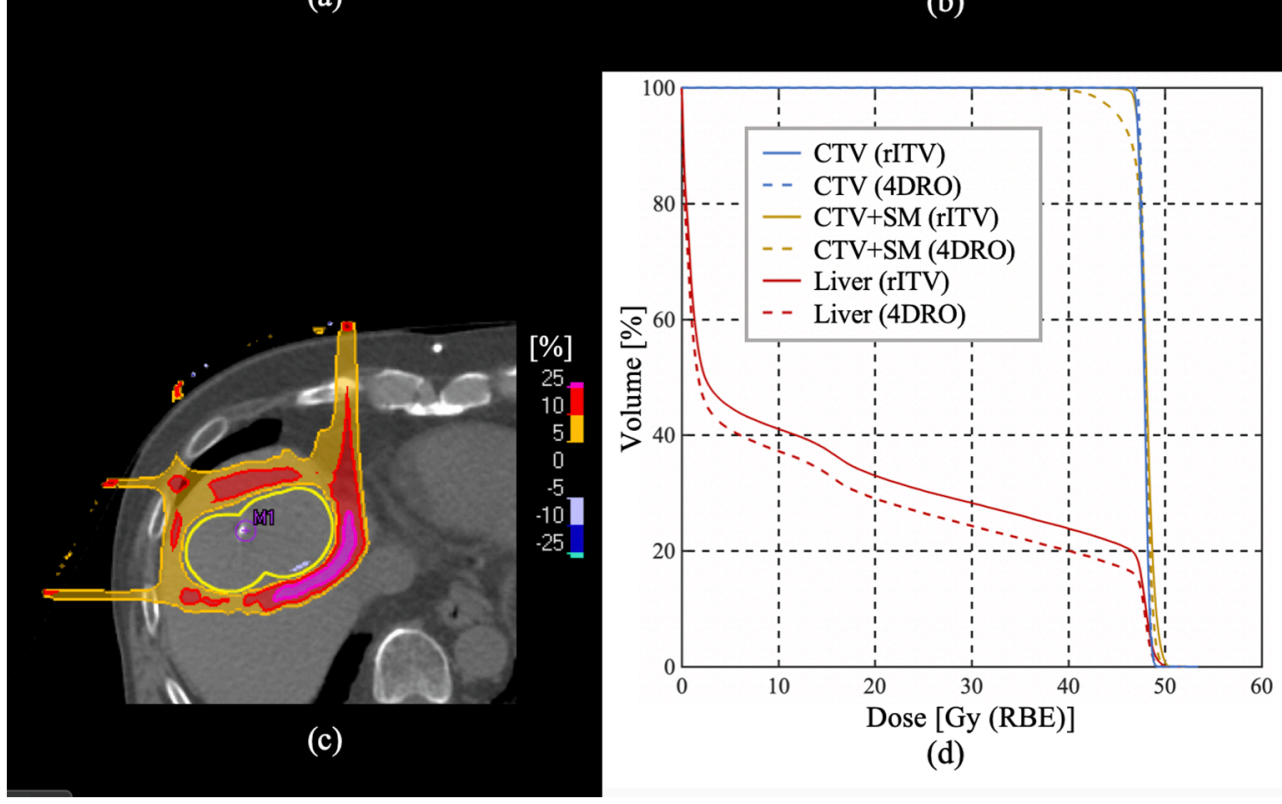

(d)
Fig. 6. Accumulated dose distributions for a lung case (patient no.18) with (a) rITV plan, (b) 4DRO plan, and (c) the dose differences (6a minus 6b). CTV is depicted as a yellow line. Red, orange, green, light blue, blue and purple lines show 95\%, 90\%, 70\%, 50\%, $30 \%$ and $10 \%$, respectively. (d) The dosevolume histogram. Abbreviations: CTV = clinical target volume, rITV $=$ range adapted internal target volume, 4DRO: 4-dimensional robust optimization. (For interpretation of the references to colour in this figure legend, the reader is referred to the web version of this article.)

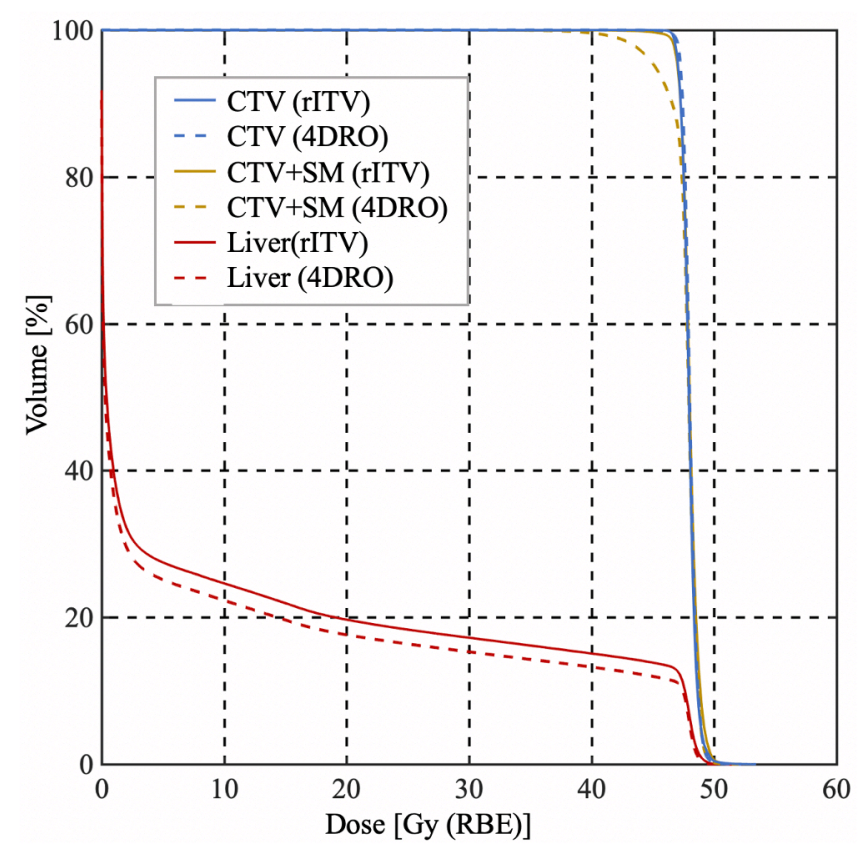

Fig. 7. Dose-volume histogram averaged over all lung cases for range-adapted ITV (solid line) and 4DRO (dotted line) treatment plans. Abbreviations: CTV = clinical target volume, $\mathrm{rTTV}=$ range adapted internal target volume, $4 \mathrm{DRO}=4$ dimensional robust optimization.
Table 5

Summary of the dose-volume parameters (Mean \pm SD) for liver cases.

\begin{tabular}{llllr}
\hline Structure & Metric & rITV plan (\%) & 4DRO plan (\%) & $P$-value* \\
\hline CTV & D95 & $98.1 \pm 0.4$ & $98.5 \pm 0.2$ & 0.001 \\
CTV + setup margin & D95 & $98.0 \pm 0.5$ & $94.1 \pm 0.9$ & $<0.001$ \\
Liver & V5 & $27.5 \pm 11.6$ & $25.1 \pm 11.3$ & $<0.001$ \\
& V10 & $24.6 \pm 11.2$ & $22.3 \pm 10.8$ & $<0.001$ \\
& V20 & $19.7 \pm 10.3$ & $17.6 \pm 9.8$ & $<0.001$ \\
& V30 & $17.2 \pm 9.7$ & $15.3 \pm 9.2$ & $<0.001$ \\
\hline
\end{tabular}

*Wilcoxon signed rank test.

Abbreviations: CTV = clinical target volume, rITV $=$ range-adapted internal target volume, $4 \mathrm{DRO}=4$-dimensional robust optimization, $\mathrm{SD}=$ standard deviation.

respiratory management, another possible approach, material override, has been reported $[24,36]$. This approach needs less computational cost and is available with many treatment planning systems. Fredriksson et al. reported its usefulness in improving target coverage, while it was very conservative and resulted in higher OAR dose than robust optimization. However, adjusting the overriding value according to WEPL information can optimize the trade-off relationship between target coverage and OAR sparing [36]. Whether optimized material override approach is more effective than rITV and robust optimization, or not, is beyond the scope of this study. It is therefore future task to thoroughly evaluate the four-dimensional planning strategy. 


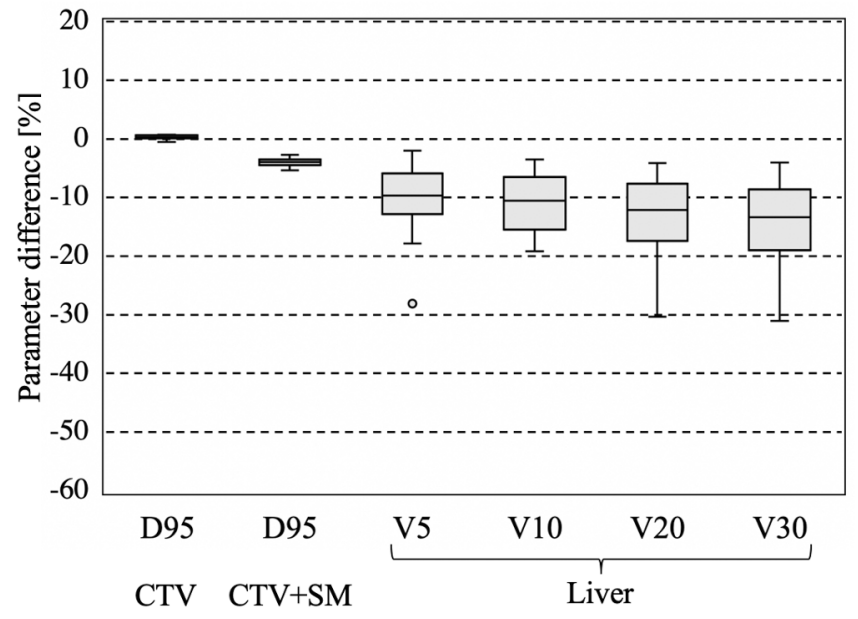

Fig. 8. A boxplot of the dose-volume parameter differences between $4 \mathrm{DRO}$ plans and rITV plans for respective VOIs in liver cases. Parameter differences were calculated according to the Eq. (1). The central horizontal line in the box indicates the median, and the bottom and top edges of the box indicate the 25th percentiles ( $q 1)$ and 75th percentiles ( $q 3)$, respectively. The whiskers extend to the most extreme data points not considered outliers. Outlier (gray circle) is identified if it is $>\boldsymbol{q} 3+(\boldsymbol{q} 3-\boldsymbol{q} 1) \times 1.5$ or $<\boldsymbol{q} 1-(\boldsymbol{q} 3-\boldsymbol{q} 1) \times 1.5$. Abbreviations: $\mathrm{CTV}=$ clinical target volume, $\mathrm{VOI}=$ volume of interest, $\mathrm{SM}=$ setup margin.

\subsection{Study limitations}

Some limitations in this study should be warranted. First, we evaluated the time accumulated dose distribution using 4DCT by applying DIR. The interplay effect between dynamic beam position and respiratory motion was not considered in this study, because this effect could be minimized by respiratory gating and phase-correlated rescanning $[18,37,38]$, which we routinely apply to treat moving targets. However, when the interplay remains due to treatment machine specifications, it should be noted that more realistic dose estimation may be possible by including this interplay.

Second, the rITV was created through intensity-based DIR implemented in the 4D-tool $[20,21]$, while 4DRO and dose accumulation were performed by a hybrid intensity-based DIR [22]. We created the CTV contours for all respiratory phases using 4DCT at T50 by applying DIR. As the generated contour accuracy could be affected by DIR accuracy, especially in low contrast anatomical sites such as liver, both certified oncologist and medical physicist carefully checked the generated contours before the analysis. Since all evaluations were performed using the same DIR algorithm for both rITV and 4DRO plans, both plans were compared fairly and DIR error would not significantly influence the results in this study.

Third, we used a CT-to-WEPL table created according to Kanematsu et al. [39] in order to create the rITV, while dose calculations were performed based on the WEPL calculation algorithm implemented in RayStation [40]. This is because the workstation for the 4DRO plan (RayStation) did not integrate a design function of rITV. The effect of the differences in CT-to-WEPL conversion would be small because both

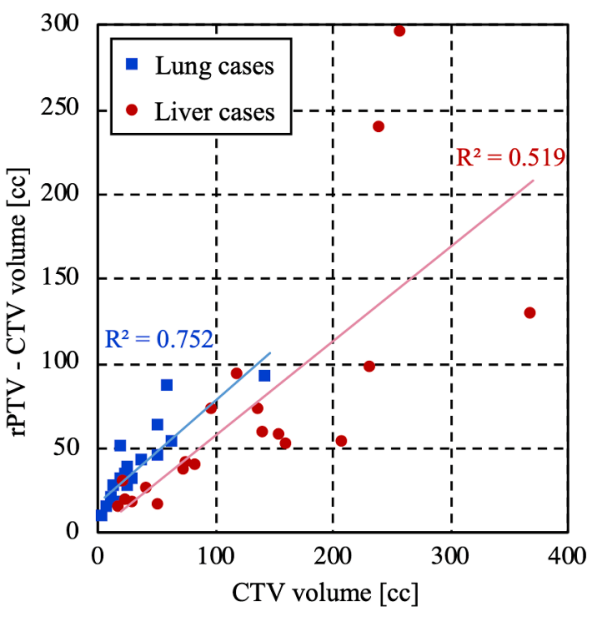

(a)

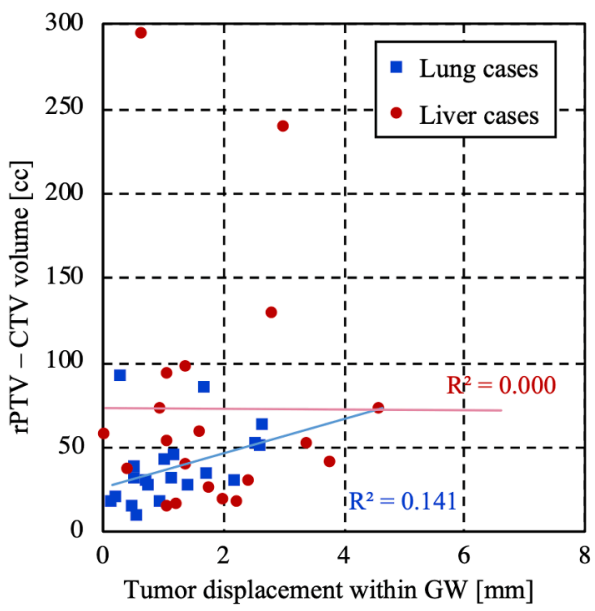

(c)

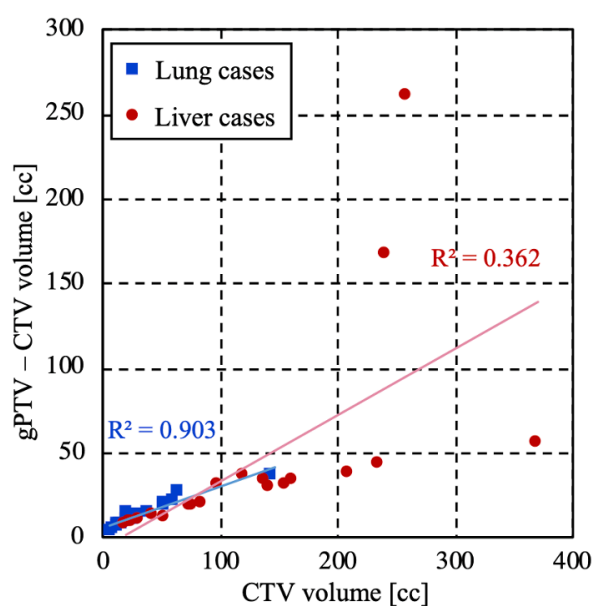

(b)

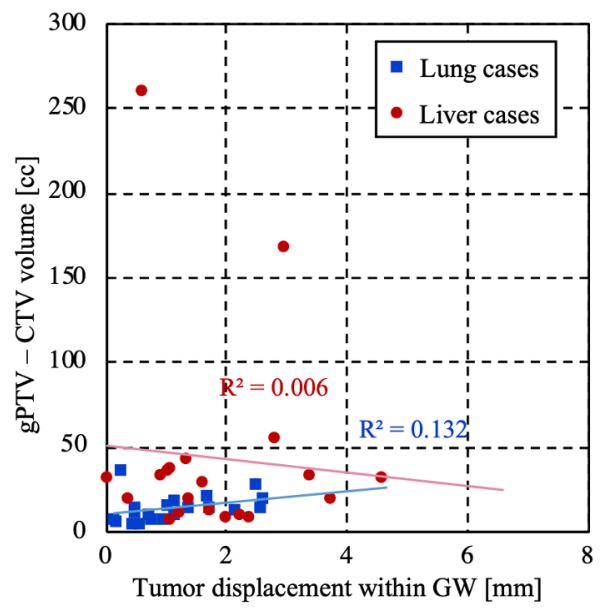

(d)
Fig. 9. Scatter plots between CTV volume and rPTV minus CTV volume (a), CTV volume and gPTV minus CTV volume (b), tumor displacement within gating window and rPTV minus CTV volume (c), tumor displacement within gating window and gPTV minus CTV volume (d) for lung and liver cancer patients. Coefficients of determination $\left(\mathrm{R}^{2}\right)$ are displayed in all figures. Abbreviations: CTV = clinical target volume, $\mathrm{rPTV}=$ range-adapted planning target volume, gPTV = geometrical planning target volume, $\mathrm{GW}=$ gating window. 
methods are based on the representative tissues reported by ICRP [41].

Forth, the uncertainties in target volume delineation and underestimation of the inter/intra-fractional errors may result in unexpected low doses to regions in the CTV, especially with the 4DRO plan. We used only one CTV for each patient and cannot estimate these uncertainties. The sensitivity of an optimized plan against these uncertainties and errors remains to be determined.

Fifth, since our main purpose of this study was to evaluate dose distributions in between 4DRO and rITV-based treatment plans, dose perturbation due to setup error was beyond of this study. However, our group previously reported about the dose perturbation due to the setup error using rITV-based treatment plan [42], and it concluded that the target coverage and excessive dose to OARs were not affected by the setup errors within setup margins. Regarding to the 4DRO, Fredriksson [24] also reported that the robust optimization did not degrade the target coverage due to the setup error.

Finally, since we used only one 4DCT dataset per patient, respiratory variability is not accounted. Respiratory variability has been reported to significantly influence the target coverage, and could be handled with probabilistic ITV approach based on four-dimensional MRI [43]. Although this approach is effective, it requires clinical resources, such as MRI acquisition over multiple respiratory cycles. We assumed that the irregular breathing can be compensated by 4DRO or rITV approach with adequate setting of setup error. It is still needed to account the effect by respiratory variability for more accurate estimation of four-dimensional dose distributions.

\section{Conclusion}

We conducted a comparison study between 4DRO and rITV plans for respiratory-gated C-PBS treatment. The 4DRO plan resulted in slightly worse target coverage and significantly better OAR sparing than the rITV plan. Although target coverage in the presence of respiratory motion and setup error was acceptable with 4DRO plan, contouring uncertainty and setup error should be carefully controlled to avoid unexpected under-dosage to the CTV when 4DRO is used.

\section{Declaration of Competing Interest}

The authors declare the following financial interests/personal relationships which may be considered as potential competing interests: RaySearch Laboratories AB provided the RayStation used for treatment planning in this study. Wataru Furuichi is an employee of Accelerator Engineering Corporation.

\section{Acknowledgments}

We thank Libby Cone, MD, MA, from DMC Corp. (www.dmed.co.jp $<$ http://www.dmed.co.jp/>) for editing drafts of this manuscript.

Funding

This research received no specific grant from any funding agency in the public, commercial, or not-for-profit sectors.

\section{References}

[1] Kamada T, Tsujii H, Blakely EA, Debus J, De Neve W, Durante M, et al. Carbon ion radiotherapy in Japan: an assessment of 20 years of clinical experience. Lancet Oncol 2015;16:e93-100.

[2] Tsujii H, Kamada T. A review of update clinical results of carbon ion radiotherapy. Jpn J Clin Oncol 2012;42:670-85.

[3] Matsumoto K, Nakamura K, Shioyama Y, Sasaki T, Ohga S, Yamaguchi T, et al Treatment planning comparison for carbon ion radiotherapy, proton therapy and intensity-modulated radiotherapy for spinal sarcoma. Anticancer Res 2015;35: 4083-9.
[4] Barendsen GW. Differences between biological effects of high LET and low LET radiations in relation to their application in radiotherapy. Radiologia Clinica 1977; 46:380-9.

[5] Bragg WH, Kleeman R. LXXIV. On the ionization curves of radium. Lond Edinb Dubl Phil Mag. 1904;8:726-38.

[6] Besserer J, de Boer J, Dellert M, Gahn C, Moosburger M, Pedroni E, et al. Rapid proton radiography with the proton-gantry of the Paul Scherrer Institute. Biomed Tech Biomed Eng 1997;42(Suppl):490-1.

[7] Combs SE, Fllerbrock M, Haberer T, Habermehl D, Hoess A, Jakel O, et al. Heidelberg Ion therapy center (HIT): initial clinical experience in the first 80 patients. Acta Oncol 2010;49:1132-40.

[8] Bert C, Grozinger SO, Rietzel E. Quantification of interplay effects of scanned particle beams and moving targets. Phys Med Biol 2008;53:2253-65.

[9] Habermehl D, Debus J, Ganten T, Ganten MK, Bauer J, Brecht IC, et al. Hypofractionated carbon ion therapy delivered with scanned ion beams for patients with hepatocellular carcinoma - feasibility and clinical response. Radiat Oncol 2013;8:59.

[10] Landberg T, Chavaudra J, Dobbs J, Gerard J-P, Hanks G, Horiot J-C, et al. Report 62: prescribing, recording and reporting photon beam therapy (Supplement to ICRU Report 50). J Int Commission Radiat Units Measurements 2016. os32:NP-NP.

[11] Knopf AC, Boye D, Lomax A, Mori S. Adequate margin definition for scanned particle therapy in the incidence of intrafractional motion. Phys Med Biol 2013;58: 6079-94.

[12] Mori S, Takei Y, Shirai T, Hara Y, Furukawa T, Inaniwa T, et al. Scanned carbon-ion beam therapy throughput over the first 7 years at national institute of radiological sciences. Phys Med 2018;52:18-26.

[13] Graeff C. Robustness of 4D-optimized scanned carbon ion beam therapy against interfractional changes in lung cancer. Radiother Oncol 2017;122:387-92.

[14] Graeff C, Durante M, Bert C. Motion mitigation in intensity modulated particle therapy by internal target volumes covering range changes. Med Phys 2012;39: 6004-13.

[15] Liu W, Liao Z, Schild SE, Liu Z, Li H, Li Y, et al. Impact of respiratory motion on worst-case scenario optimized intensity modulated proton therapy for lung cancers. Pract Radiat Oncol 2015;5:e77-86.

[16] Pflugfelder D, Wilkens JJ, Oelfke U. Worst case optimization: a method to account for uncertainties in the optimization of intensity modulated proton therapy. Phys Med Biol 2008;53:1689-700.

[17] Stuschke M, Kaiser A, Pottgen C, Lubcke W, Farr J. Potentials of robust intensity modulated scanning proton plans for locally advanced lung cancer in comparison to intensity modulated photon plans. Radiother Oncol 2012;104:45-51.

[18] Mori S, Inaniwa T, Furukawa T, Takahashi W, Nakajima M, Shirai T, et al. Amplitude-based gated phase-controlled rescanning in carbon-ion scanning beam treatment planning under irregular breathing conditions using lung and liver 4DCTs. J Radiat Res 2014;55:948-58.

[19] Mori S, Karube M, Shirai T, Tajiri M, Takekoshi T, Miki K, et al. Carbon-ion pencil beam scanning treatment with gated markerless tumor tracking: an analysis of positional accuracy. Int J Radiat Oncol Biol Phys 2016;95:258-66.

[20] Sharp GC, Kandasamy N, Singh H, Folkert M. GPU-based streaming architectures for fast cone-beam CT image reconstruction and demons deformable registration. Phys Med Biol 2007;52:5771-83.

[21] Mori S, Inaniwa T, Miki K, Shirai T, Noda K. Implementation of a target volume design function for intrafractional range variation in a particle beam treatment planning system. Br J Radiol 2014;87:20140233.

[22] Samavati N, Velec M, Brock K. A hybrid biomechanical intensity based deformable image registration of lung 4DCT. Phys Med Biol 2015;60:3359-73.

[23] National Electrical Manufacturers Association (NEMA). DICOM ${ }^{\circledR}$ (Digital Imaging and Communications in Medicine).

[24] Fredriksson A, Forsgren A, Hardemark B. Minimax optimization for handling range and setup uncertainties in proton therapy. Med Phys 2011;38:1672-84.

[25] Inaniwa T, Furukawa T, Kase Y, Matsufuji N, Toshito T, Matsumoto Y, et al. Treatment planning for a scanned carbon beam with a modified microdosimetric kinetic model. Phys Med Biol 2010;55:6721-37.

[26] Hayashi K, Yamamoto N, Karube M, Nakajima M, Matsufuji N, Tsuji H, et al. Prognostic analysis of radiation pneumonitis: carbon-ion radiotherapy in patients with locally advanced lung cancer. Radiat Oncol 2017;12:91.

[27] Ono T, Nakamura T, Yamaguchi H, Azami Y, Takayama K, Suzuki M, et al. Clinical results of proton beam therapy for elderly patients with non-small cell lung cancer. Radiation Oncology 2018;13:19.

[28] Tsujino K, Hashimoto T, Shimada T, Yoden E, Fujii O, Ota Y, et al. Combined analysis of V20, VS5, pulmonary fibrosis score on baseline computed tomography, and patient age improves prediction of severe radiation pneumonitis after concurrent chemoradiotherapy for locally advanced non-small-cell lung cancer. J Thorac Oncol 2014;9:983-90.

[29] Cheng JC, Wu JK, Huang CM, Huang DY, Cheng SH, Lin YM, et al. Radiationinduced liver disease after radiotherapy for hepatocellular carcinoma: clinical manifestation and dosimetric description. Radiother Oncol 2002;63:41-5.

[30] Mizumoto M, Okumura T, Hashimoto T, Fukuda K, Oshiro Y, Fukumitsu N, et al. Evaluation of liver function after proton beam therapy for hepatocellular carcinoma. Int J Radiat Oncol Biol Phys 2012;82:e529-35.

[31] Takahashi W, Nakajima M, Yamamoto N, Yamashita H, Nakagawa K, Miyamoto T, et al. A prospective nonrandomized phase I/II study of carbon ion radiotherapy in a favorable subset of locally advanced non-small cell lung cancer (NSCLC). Cancer 2015;121:1321-7.

[32] Shibuya K, Ohno T, Terashima K, Toyama S, Yasuda S, Tsuji H, et al. Short-course carbon-ion radiotherapy for hepatocellular carcinoma: a multi-institutional retrospective study. Liver Int 2018;38:2239-47. 
[33] Steitz J, Naumann P, Ulrich S, Haefner MF, Sterzing F, Oelfke U, et al. Worst case optimization for interfractional motion mitigation in carbon ion therapy of pancreatic cancer. Radiat Oncol 2016;11:134.

[34] Lin L, Kang M, Huang S, Mayer R, Thomas A, Solberg TD, et al. Beam-specific planning target volumes incorporating 4D CT for pencil beam scanning proton therapy of thoracic tumors. J Appl Clin Med Phys 2015;16:5678.

[35] Lin L, Souris K, Kang M, Glick A, Lin H, Huang S, et al. Evaluation of motion mitigation using abdominal compression in the clinical implementation of pencil beam scanning proton therapy of liver tumors. Med Phys 2017;44:703-12.

[36] Botas P, Grassberger C, Sharp G, Paganetti H. Density overwrites of internal tumor volumes in intensity modulated proton therapy plans for mobile lung tumors. Phys Med Biol 2018;63:035023.

[37] Mori S, Furukawa T. Rapid phase-correlated rescanning irradiation improves treatment time in carbon-ion scanning beam treatment under irregular breathing. Phys Med Biol 2016;61:3857-66.
[38] Furukawa T, Inaniwa T, Sato S, Shirai T, Mori S, Takeshita E, et al. Moving target irradiation with fast rescanning and gating in particle therapy. Med Phys 2010;37: 4874-9.

[39] Kanematsu N, Mori S, Inaniwa T. Revision of calibration method of CT-number to stopping-power-ratio conversion for treatment planning of particle therapy. Igaku Butsuri 2017;37:34-7.

[40] Taasti VT, Bäumer C, Dahlgren CV, Deisher AJ, Ellerbrock M, Free J, et al. Intercentre variability of CT-based stopping-power prediction in particle therapy: survey-based evaluation. Phys Imag Radiat Oncol 2018;6:25-30.

[41] ICRP. Adult reference computational phantoms. Ann ICRP 2009;39:1-164.

[42] He P, Mori S. Perturbation analysis of 4D dose distribution for scanned carbon-ion beam radiotherapy. Phys Med 2020;74:74-82.

[43] Krieger M, Giger A, Salomir R, Bieri O, Celicanin Z, Cattin PC, et al. Impact of internal target volume definition for pencil beam scanned proton treatment planning in the presence of respiratory motion variability for lung cancer: a proof of concept. Radiother Oncol 2020;145:154-61. 